\title{
Review of Upper Extremity Nerve Transfer in Cervical Spinal Cord Injury
}

\author{
Sarah A. Cain ${ }^{1}$ Andreas Gohritz ${ }^{2,3}$ Jan Fridén ${ }^{2,4}$ Natasha van Zyl $\left.\right|^{1,5,6}$
}

${ }^{1}$ Department of Plastic and Reconstructive Surgery, The Royal

Melbourne Hospital, Parkville, Victoria, Australia

2 Tetraplegia Hand Surgery, Swiss Paraplegia Centre, Nottwil, Switzerland

${ }^{3}$ Department of Plastic, Reconstructive and Aesthetic Surgery, Hand Surgery, University Hospital, Basel, Switzerland

${ }^{4}$ Center of Advanced Reconstruction of Extremities (CARE), National

Reference Center for Tetraplegia Surgery, Sahlgrenska University

Hospital, Göteborg, Sweden

${ }^{5}$ Victorian Spinal Cord Service, Austin Health, Heidelberg,

Victoria, Australia

${ }^{6}$ Department of Medicine, University of Melbourne, Parkville,

Victoria, Australia

J Brachial Plex Peripher Nerve Inj 2015;10:e34-e42.

\begin{abstract}
Address for correspondence Natasha van Zyl, MBBS, Department of Plastic and Reconstructive Surgery, Austin Health, 145 Studley Road, Heidelberg, VIC 3084, Australia (e-mail: info@natashavanzyl.com.au).
\end{abstract}

\begin{abstract}
Keywords

- nerve transfer

- tetraplegia

- spinal cord injury

- quadriplegia

Objective Several nerve transfers have now been successfully performed for upper limb reanimation in tetraplegia. This study was performed to review the use of nerve transfers for upper limb reanimation in tetraplegia.

Methods Medline and Embase (1950 to February 11, 2015) were searched using a search strategy designed to include any studies that reported cases of nerve transfer in persons with cervical spinal cord injury $(\mathrm{SCl})$.

Results A total of 103 manuscripts were selected initially and full-text analysis produced 13 studies with extractable data. Of these manuscripts, 10 reported single cases and 3 reported case series. Eighty-nine nerve transfers have been performed in 57 males and 2 females with a mean age of 34 years. The mean $\mathrm{SCl}$ level was $\mathrm{C} 6$ (range: $\mathrm{C5}$ 7), time to surgery post-SCI was 19.9 months (range: 4.1-156 months), and follow-up time was 18.2 months (range: 3-60 months). All case reports recorded a Medical Research Council (MRC) score of 3 or 4 for recipient muscle power, but two early case series reported more variable results.

Conclusion This review documents the current status of nerve transfer surgery for upper limb reanimation in tetraplegia and summarizes the functional results in 59 cases with 89 nerve transfers performed, including 15 cases of double-nerve transfer and 1 case of triple-nerve transfer.
\end{abstract}

\section{Introduction}

The estimated global incidence of spinal cord injury (SCI) is 40 to 80 new cases per million population per year, which means that every year 250,000 to 500,000 people worldwide become paralyzed. Most of these are young, healthy, and active in their most productive years between the second and fourth decades of life. More than $50 \%$ of all SCIs occur at the cervical level and lead to tetraplegia. ${ }^{1}$

There are many challenges to be faced. The upper extremity remains, apart from the brain, the most important functional resource of the patient with tetraplegia. Upper received

March 9, 2015

accepted

June 3, 2015

published online

August 6, 2015
DOI http://dx.doi.org/

10.1055/s-0035-1558427.

ISSN $1749-7221$.
Copyright $\odot 2015$ Georg Thieme Verlag

KG Stuttgart · New York
License terms

(@) (1) $\circledast$ 
extremity usability is ranked to be the most desirable ability to regain after cervical SCI. It is given a higher priority than bowel, bladder, sexual function, or walking ability by patients and their caregivers alike. ${ }^{2}$ Surgical improvement in arm and hand function therefore has a huge impact on independence, mobility, and quality of life.

Until recently, tendon transfers have been the workhorse of upper extremity reconstruction in tetraplegia. ${ }^{3}$ Tendon transfers move a functioning muscle to a new insertion site to recreate the function of a paralyzed muscle. In contrast, nerve transfers allow direct reanimation of a paralyzed muscle, thus preserving its biomechanical functional advantage. Nerve transfers have the potential to reconstruct more than one function for a single-nerve transfer and to avoid the technical challenges of tendon transfer surgery such as tendon tensioning, mechanical failure (stretch or rupture) of transfers over time, adhesion formation, and the longer postoperative immobilization or splinting required post-tendon transfer.

Nerve transfers are a well-established surgical technique for reanimating paralyzed muscles in brachial plexus and peripheral nerve injury. ${ }^{4}$ Patients reliably attain muscle power against gravity and often against resistance. ${ }^{4-6}$ The use of nerve transfers to restore upper limb function in tetraplegic patients is not a new concept. Benassy in 1965 described the first case, a musculocutaneous nerve to median nerve transfer, in $1962 .^{7}$ Kiwerski in $1982^{8}$ reported 20 cases and later went on to report further results in $1991 .{ }^{9}$ However, despite these early reported cases, the use of nerve transfers for the reconstruction of upper limb function in tetraplegia has received little attention until relatively recently. This review documents the current status of nerve transfer surgery for upper limb reanimation in tetraplegia, and summarizes the reported outcome of 90 transfers, including 15 case reports of double-nerve transfer and one case of triple-nerve transfer.

\section{Methods}

A review of the published literature was performed for the primary outcome of nerve transfer in patients with tetraplegia for restoration of upper limb function. A Preferred Reporting Items for Systematic Reviews and Meta-Analyses (PRISMA) (<www.prisma-statement.org $>$ ) style was adhered to where possible, but quality assessment was not performed, as the target study type was case series and case reports.

\section{Eligibility Criteria}

All English language manuscripts reporting original data on patients with cervical SCI undergoing nerve transfer for restoration of upper limb function were included. Nerve transfers performed on patients without SCI were excluded. Case series, case-control studies, cohort studies, and randomized controlled trials were included.

\section{Search Criteria}

Medline and Embase databases were searched from 1950 to February 11, 2015. The Cochrane Collaboration database and the National Health Service (NHS) Evidence Health Information Resources Web site were also searched. In addition, a manual reference check of the retrieved articles to identify additional studies not captured in the original search was performed. No unpublished trials were included. We designed a search strategy to include manuscripts relevant to any case of nerve transfer in the upper limb to restore function in a patient with cervical SCI. The following keywords were used in the search strategy: Tetraplegia, Quadriplegia, Nerve Transfer, and Spinal Cord Injury.

Once the searches had been completed, studies were selected in an unblinded standardized manner. The abstracts were reviewed and articles unrelated to our topic and duplicate articles were excluded ( $\mathbf{- F i g . 1}$ ). The abstracts were then reviewed to ascertain whether they met the described inclusion criteria.

\section{Data Extraction and Statistical Analysis}

Following formal article review, data representing patient demographics, country of origin, mean time to surgery, mean follow-up time, and nerve transfer used were extracted. Functional outcomes were also extracted where reported. Kiwerski reported outcomes for musculocutaneous to median nerve transfers in two reports. ${ }^{8,9}$ The 20 patients from the first study were included again in the second. Quantitative assessments were performed primarily with descriptive data. Case-by-case analysis was performed for summary data.

\section{Results}

\section{Search Results}

Thirteen journal articles reported 89 nerve transfers in the upper limb in 59 patients with tetraplegia, including 15 cases of dual-nerve transfer ${ }^{10-12}$ and 1 case of triple-nerve transfer. ${ }^{13}$ All cases were single-case reports except the early series reported by Kiwerski and Krasuski ${ }^{8,9}$ and the most recently published series by Bertelli. The studies are summarized in - Table 1. There were 57 males and 2 females with a mean age of 34 years (range: $17-71$ years). The mean spinal cord injury ( $\mathrm{SCI}$ ) level was $\mathrm{C6}$ (range: $\mathrm{C5}-7$ ). The mean time delay between SCI and surgery was 19.9 months (range: 1156 months). The mean follow-up time was 18.2 months (range: 3-60 months). All case reports of nerve transfer in a patient with tetraplegia reported a Medical Research Council (MRC) score of 3 or 4 for recipient muscle power, but the two earlier case series studies showed a range of results ( - Table 1). A summary of nerve transfers used is presented in -Table 2.

\section{Summary of Cases}

Benassy and Robert first used the musculocutaneous nerve to restore median nerve function in tetraplegia in 1962. The transfer proved "very useful" for the patient and allowed him to type with the left hand, eat unaided (except for cutting meat), light a match, and drive his wheelchair. He achieved M3 power to pronator teres, flexor carpi radialis (FCR), and flexor digitorum profundus (FDP) to the index and middle fingers and M2 power to flexor pollicis longus (FPL). ${ }^{7}$ An early 


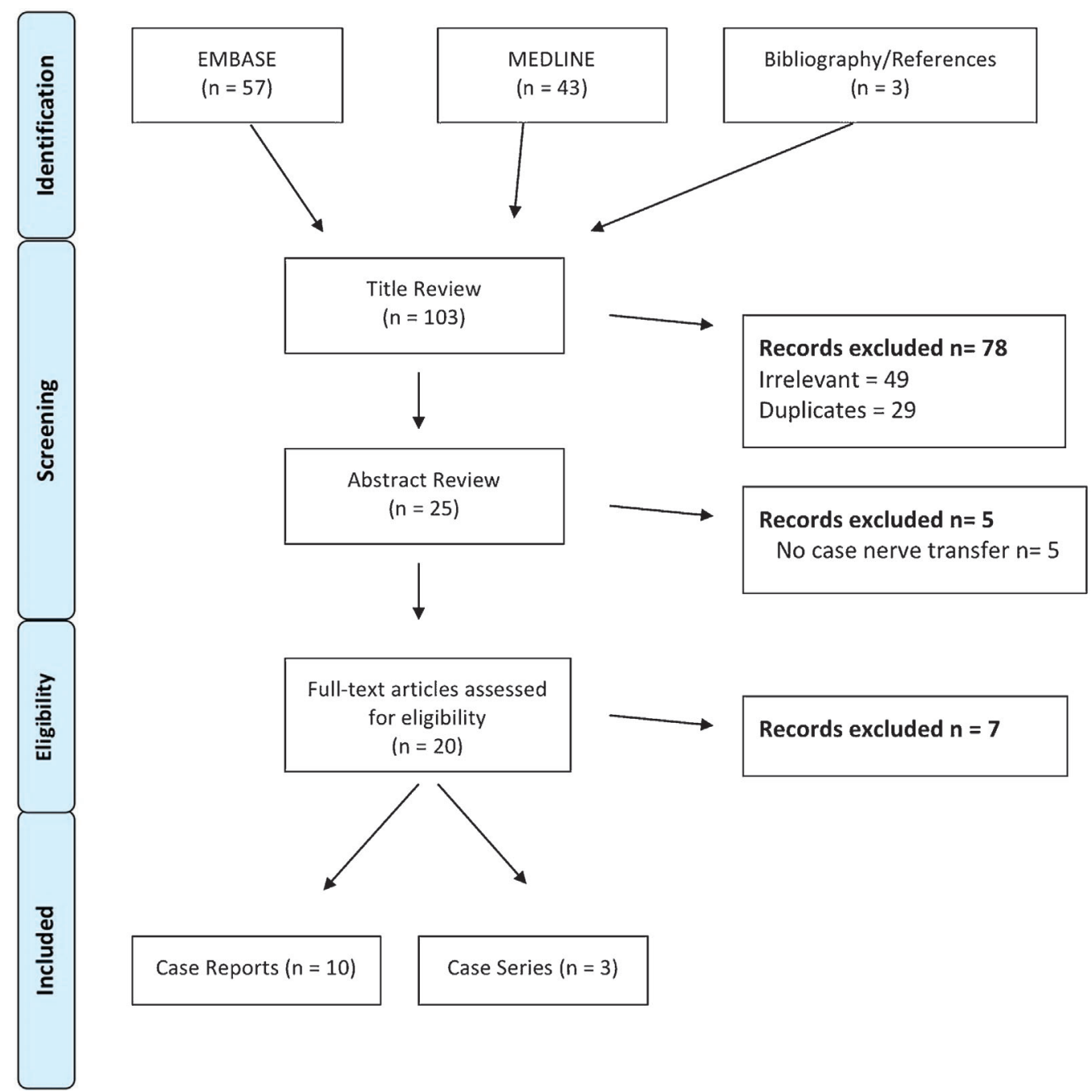

Fig. 1 Preferred Reporting Items for Systematic Reviews and Meta-Analyses (PRISMA) flow chart of search results.

case series, published by Kiwerski in $1982,{ }^{8}$ reported on 20 patients with a C6-7 SCI who underwent a musculocutaneous to median nerve transfer 1 to 10 months after SCI. All but one patient were males with a mean age of 25.2 years. Results were reported as follows: "good" in nine patients, "satisfactory" in six, and "poor" in five patients. The result was "good" if functional grasp was restored, "satisfactory" if grasp was weak but still useful, and "bad" if no result was attained. Krasuski and Kiwerski went on to report a total of 42 patients with C6-7 complete SCI who underwent a musculocutaneous to median nerve transfer an average of 3 to 4 months post injury. ${ }^{9}$ Results were similarly reported on a three-point grading of simple hand function. "Good," "fair," and "bad" results were seen in 16,16 , and 10 patients, respectively. There was an association, which was not statistically tested, between younger age at surgery and better results. There was also a relationship between shorter time lapse from injury to surgery and better outcomes, with 10 of the 16 patients with "good" results undergoing surgery within 3 months of injury.

Bertelli et al have significantly contributed to the published work on nerve transfers in tetraplegia over the last 3 years. ${ }^{11,14-17}$ The first published case report from this group presented a supinator nerve to posterior interosseous nerve (PIN) transfer, performed bilaterally, 7 months after an SCI. ${ }^{17}$ The authors reported that 6 months after surgery, with the wrist in neutral, extension of the thumb and finger was "almost full," bilaterally. The strength of the metacarpophalangeal extension scored M4, and hand aperture was almost complete. This group went on to publish a laboratory anatomical study, which used eight formalin fixed and two fresh cadavers to examine the surgical feasibility of transfer of axillary nerve branches to reconstruct elbow extension. ${ }^{18}$ Their conclusion stated that teres minor and posterior deltoid nerve branches could be transferred to nerves to the long and/ or upper medial heads of triceps and/or to the thoracodorsal nerve. They subsequently published a case of a 21-year-old man, 9 months post-SCI who had triceps successfully reanimated to an M4 power grade bilaterally using a teres minor nerve to long head of triceps nerve transfer. ${ }^{15}$

Bertelli et al have extended their series with three further case reports. A 53-year-old man, 5 months after incomplete tetraplegia (central cord syndrome), had a brachialis nerve to triceps nerve transfer. This patient was reported to have M4 and $5 \mathrm{~kg}$ triceps strength 12 months postoperatively. ${ }^{14}$ A 24- 
Nerve Transfer in Tetraplegia Cain et al. e37

\begin{tabular}{|c|c|c|c|c|c|c|c|c|c|c|c|c|c|}
\hline 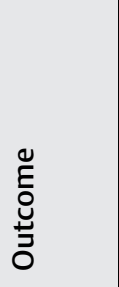 & 1 & $\begin{array}{l}n \\
\infty \\
6 \\
\sim \\
\sigma \\
\cup\end{array}$ & $\begin{array}{l}0 \\
0 \\
0 \\
0 \\
\frac{1}{6} \\
6 \\
0\end{array}$ & 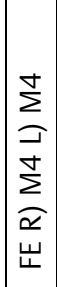 & 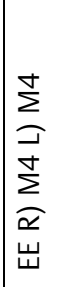 & 1 & 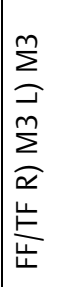 & $\sum_{\mu}^{J}$ & 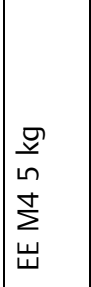 & $\sum_{\stackrel{m}{M}}^{m}$ & 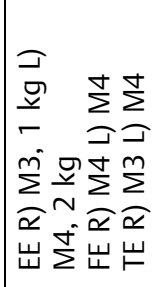 & 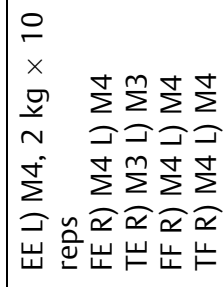 & 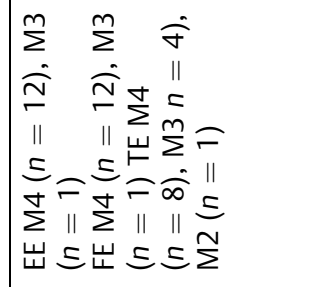 \\
\hline 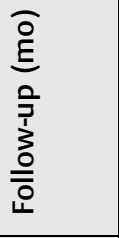 & $\stackrel{\nabla}{N}$ & 8 & 1 & 6 & $\stackrel{\nabla}{\sim}$ & $m$ & $\stackrel{\operatorname{Ln}}{\longrightarrow}$ & $\stackrel{\nabla}{\mp}$ & $\stackrel{m}{r}$ & in & $\approx$ & 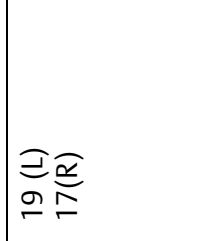 & $\stackrel{\circ}{\longleftarrow}$ \\
\hline 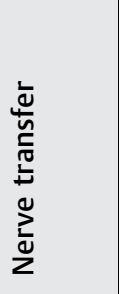 & 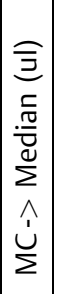 & 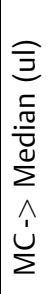 & 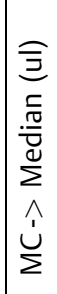 & 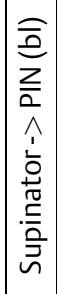 & 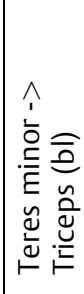 & 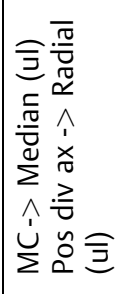 & 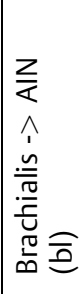 & 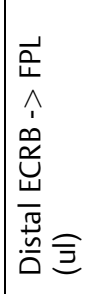 & 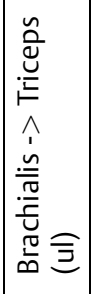 & 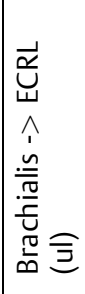 & 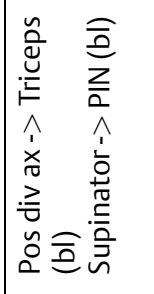 & 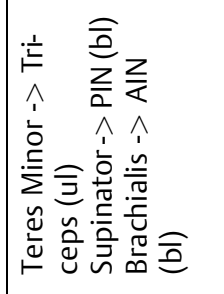 & 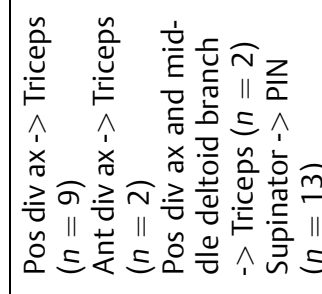 \\
\hline 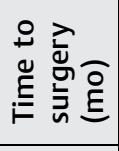 & $\mp$ & $\begin{array}{l}\infty \\
\dot{+} \\
\end{array}$ & 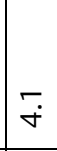 & $\wedge$ & $\sigma$ & L̊ & $\stackrel{\sim}{\sim}$ & $r$ & in & $\stackrel{\simeq}{\simeq}$ & $\stackrel{\infty}{-}$ & 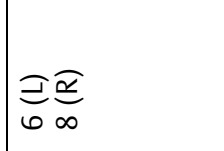 & $\wedge$ \\
\hline 这莣 & 1 & 1 & 1 & 1 & 1 & $\infty$ & $<$ & 1 & 1 & 1 & 1 & 1 & 1 \\
\hline $\begin{array}{l}\text { 气̄ } \\
\text { 늠 } \\
\underline{u}\end{array}$ & 1 & & 1 & 1 & $\sim$ & $\sim$ & in & 1 & 1 & 1 & $m$ & 1 & 1 \\
\hline 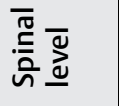 & \begin{tabular}{|l|}
0 \\
1 \\
$u$ \\
.
\end{tabular} & $\begin{array}{l}0 \\
\vdots \\
0\end{array}$ & $\begin{array}{l}1 \\
b \\
\vdots\end{array}$ & 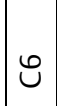 & ( & ט & O & 1 & 1 & O & $\bar{U}$ & 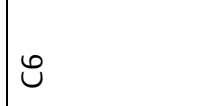 & U \\
\hline 岁 & $\hat{m}$ & $\stackrel{\sim}{\sim}$ & $\stackrel{\circ}{7}$ & $\stackrel{i}{i}$ & $\bar{\sim}$ & $\stackrel{\infty}{\sim}$ & $\pi$ & $\stackrel{d}{\sim}$ & nิ & p & શి & $\bar{\tau}$ & $\stackrel{\varpi}{\sim}$ \\
\hline ڤ & $\mid \begin{array}{l}\Sigma \\
-\end{array}$ & $\begin{array}{l}\Sigma \\
\sigma \\
\square \\
5 \\
-\end{array}$ & $\begin{array}{l}\Sigma \\
\bar{\sigma} \\
\longleftarrow= \\
-\end{array}$ & $\Sigma$ & $\Sigma$ & $\Sigma$ & $\Sigma$ & $\Sigma$ & $\Sigma$ & $\Sigma$ & $\Sigma$ & $\Sigma$ & $\begin{array}{l}\sum \\
0 \\
4 \\
-\end{array}$ \\
\hline$z$ & - & 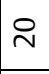 & $\stackrel{\stackrel{\sim}{*}}{ }$ & - & - & - & - & - & - & - & - & - & $\wedge$ \\
\hline $\begin{array}{l}\stackrel{Z}{E} \\
\text { ¿ }\end{array}$ & 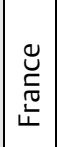 & $\begin{array}{l}\frac{0}{\mathrm{C}} \\
\frac{\pi}{0} \\
0\end{array}$ & $\begin{array}{l}\frac{7}{\mathrm{C}} \\
\frac{\mathrm{U}}{0} \\
\mathrm{O}\end{array}$ & $\begin{array}{l}\overline{\bar{N}} \\
\frac{\tilde{D}}{0} \\
\bar{D}\end{array}$ & \begin{tabular}{|l}
$\overline{\bar{N}}$ \\
$\stackrel{\mathbb{N}}{0}$ \\
0
\end{tabular} & 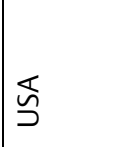 & 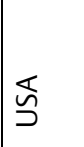 & $\begin{array}{l}\overline{\bar{N}} \\
\frac{D}{0} \\
\text { D. }\end{array}$ & $\begin{array}{l}\overline{\bar{N}} \\
\frac{D}{D} \\
\text { D. }\end{array}$ & 离 & \begin{tabular}{|l}
$\overline{\bar{N}}$ \\
$\underline{D}$ \\
D.
\end{tabular} & 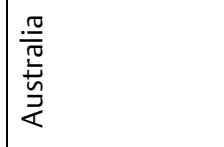 & \begin{tabular}{|l}
$\overline{\bar{N}}$ \\
$\stackrel{\mathbb{N}}{0}$ \\
D.
\end{tabular} \\
\hline 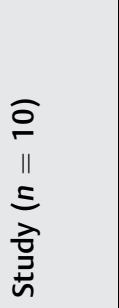 & 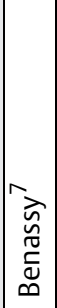 & 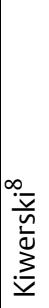 & 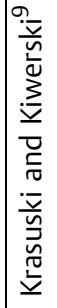 & 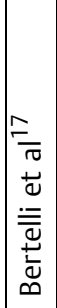 & 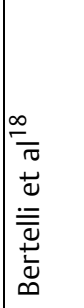 & $\mid$ & 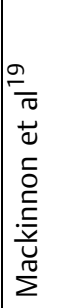 & 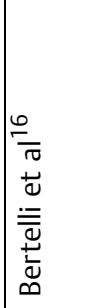 & 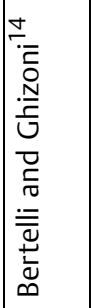 & 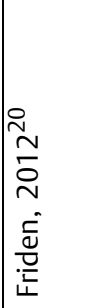 & 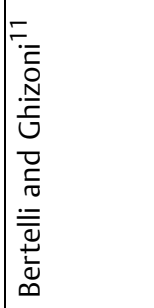 & 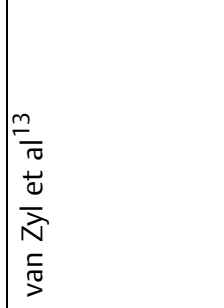 & 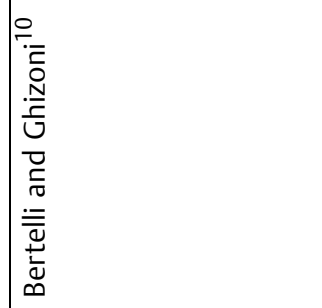 \\
\hline
\end{tabular}




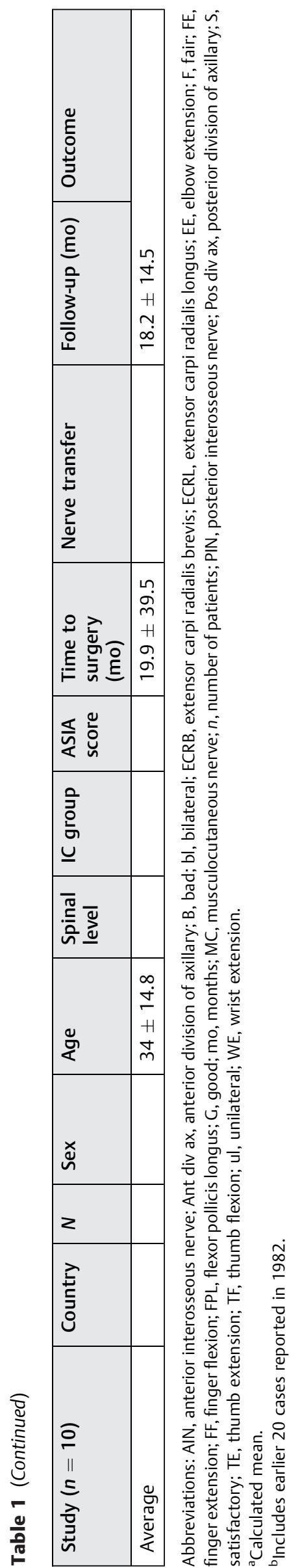

year-old man with SCI, 7 months post injury had an extensor carpi radialis brevis (ECRB) nerve to FPL nerve transfer in conjunction with a brachialis muscle to FDP tendon transfer via a tibialis anterior graft. ${ }^{16}$ An anatomical study, using five formalin-fixed and two fresh cadavers, supported the surgical feasibility of this case. At 14 months post surgery the patient was reported to have restored pinch and grasp, which measured $2 \mathrm{~kg}$ and $8 \mathrm{~kg}$, respectively. Last, a 39-year-old tetraplegic patient had bilateral surgery, again using a combination of nerve and tendon transfers, 18 months after $\mathrm{SCI}$. A selected portion of the axillary nerve (motor branch to middle deltoid on the right and the entire posterior motor division on the left) to long and medial heads of triceps nerves and a supinator to PIN transfer were performed in conjunction with a brachioradialis tendon transfer to the FPL and flexor digitorum superficialis (FDS) of the index finger. ${ }^{11}$ Twenty-two months postoperatively, triceps strength scored M3 bilaterally and metacarpophalangeal joint extension scored M4 bilaterally. The tendon transfer yielded a $1.5-\mathrm{kg}$ pinch on the left and 2-kg pinch on the right.

Most recently, Bertelli and Ghizoni's group published their first case series that included seven patients: six males and one female with 27 nerve transfers. ${ }^{10}$ The average age was 26 (range: 20-39) and surgical reconstruction was undertaken on average 7 months post-SCI. All patients had a complete C6 SCI level. All seven patients had supinator to PIN transferred to restore thumb and finger extension. Elbow extension reconstruction was achieved with nine posterior division of axillary to triceps transfers and two posterior division of axillary with a branch of middle deltoid to triceps transfers. The further two transfers performed bilaterally in the same patient were achieved by using the anterior branch of axillary to triceps. Follow-up, at mean 19 months postoperation, found M4 elbow extension in 11 upper limbs. In two upper limbs in the same patient, elbow extension scored M3. Full thumb extension scoring M4 was observed in eight upper limbs, $\mathrm{M} 3$ in four upper limbs, and M2 in one upper limb. Full metacarpal extension was M4 in 12 upper limbs and M3 in the remaining 1 upper limb. Active extension at the proximal interphalangeal joint was observed in only six hands.

Aside from the work by Bertelli et al, this review found four other case reports in the English literature. A 71-year-old man, 22 months post- $\mathrm{C} 7$ complete $\mathrm{SCI}$, underwent a bilateral brachialis to anterior interosseous nerve (AIN) transfer. Fifteen months after surgery, the patient had M3 strength for FPL and FDP. ${ }^{19}$ A 28 -year-old man with C5 tetraplegia (ASIA B) underwent a posterior division of axillary nerve (one of two fascicles contributing to the posterior deltoid) to radial and a musculocutaneous to median nerve transfer 13 years postSCI. ${ }^{12}$ Follow-up for this patient was only reported at the 3month mark with the intention to report long-term follow-up at a later date (yet to be published). Elbow flexion and shoulder flexion and abduction were noted to be M5 posttransfer. ${ }^{12}$ A 36-year-old man with incomplete C5 tetraplegia underwent a brachialis nerve to extensor carpi radialis longus (ECRL) nerve transfer 12 months post-SCI. ${ }^{20}$ The patient achieved M3 wrist extension by 5 months, allowing a tenodesis pinch, grasp, and release. 
Table 2 Summary of total nerve transfers performed in SCI

\begin{tabular}{|l|l|l|}
\hline Donor nerve/branch & Recipient nerve/branch & Number of transfers \\
\hline Posterior/middle deltoid branch & Triceps & 3 \\
\hline Posterior division of axillary & Radial & 1 \\
\hline Posterior division of axillary & Triceps & 10 \\
\hline Anterior division of axillary & Triceps & 2 \\
\hline Supinator & PIN & 19 \\
\hline Distal ECRB & FPL portion of AIN & 1 \\
\hline Brachialis & AIN & 4 \\
\hline Brachialis & ECRL & 1 \\
\hline Musculocutaneous & Median & 44 \\
\hline Teres minor & Triceps & 3 \\
\hline Brachialis & Triceps & 1 \\
\hline TOTAL & & 89 \\
\hline
\end{tabular}

Abbreviations: AIN, anterior interosseous nerve; ECRB, extensor carpi radialis brevis; ECRL, extensor carpi radialis longus; FPL, flexor pollicis longus; $\mathrm{PIN}$, posterior interosseous nerve; $\mathrm{SCl}$, spinal cord injury.

Van Zyl et al published a case of triple-nerve transfer to restore elbow extension, finger and thumb flexion, and finger and thumb extension simultaneously. Three nerve transfers were performed on the left upper limb 6 months after injury, a teres minor nerve to long head of triceps nerve, a brachialis nerve to AIN, and supinator nerve to the PIN. Two months later, only the latter two transfers were performed contralaterally, as the triceps function was adequate on that side. ${ }^{13}$ Nineteen months post surgery the patient achieved M4 elbow extension on the left, M4 thumb and finger flexion bilaterally, and M3 thumb and M4 finger extension bilaterally. Lateral pinch was $0.9 \mathrm{~kg}$ on the right and $0.72 \mathrm{~kg}$ on the left. Grasp strength measured $7 \mathrm{~kg}$ on the right and $3 \mathrm{~kg}$ on the left.

\section{Discussion}

Several nerve transfers have now been successfully used for upper extremity reanimation in tetraplegia. The musculocutaneous to either the whole median nerve or only the AIN and supinator to PIN nerve transfers have been most commonly used. It has to be emphasized that in the great majority of cases listed in this review, the authors transferred the brachialis branch onto the whole median nerve with likely loss of motor axons down sensory pathways.

- Table 3 summarizes the published nerve transfers in tetraplegia to date. As the focus shifts to nerve transfers as a potential surgical solution in tetraplegia, other transfer options will become apparent. Anatomical studies, to determine all potential appropriate donor nerves for specific functional deficits in tetraplegia, are a prerequisite for their clinical application.

\section{Timing}

The appropriate timing for surgery after SCI may be an important factor in optimizing functional outcomes in nerve transfer surgery. In tendon transfer surgery for complete SCI, it is generally recommended that surgery be delayed to at least 1 year post injury to allow time for spontaneous recovery. ${ }^{21}$ However, outcomes for tendon transfer surgery are not as time dependent as they are for nerve transfer surgery and remain feasible even decades after the original SCI.

Traditional teaching tells us that in $\mathrm{SCI}$, it is possible to separate muscles of the upper limb into three types. The first group comprises functional muscles under voluntary control, which are innervated by the supralesional segment of the spinal cord. The second includes muscles innervated by neurons at the level of the lesion. Damage to these anterior horn cells results in a lower motor neuron (LMN) denervation of these muscles. The third are paralyzed muscles, which are innervated by the infralesional segment. Preservation of the anterior horn cells results in an upper motor neuron (UMN) paralysis of these muscles. ${ }^{22}$ The nerves to the first group of muscles represent potential donor nerves. The nerves to the latter two groups are potential recipients for nerve transfer surgery. Early time to surgery is especially relevant to outcomes in the denervated muscle group, as neuromuscular end plate degeneration will eventually exclude the possibility of reanimation with new axons. However, if the muscle is paralyzed through an UMN lesion, neuromuscular degeneration will likely be slowed and this may extend the time post injury for successful reanimation with nerve transfers. Further work needs to be done to examine the histopathology of neuromuscular degeneration in UMN-injured nerves and muscle.

With the exception of one case, done at 156 months but with no result reported, all the cases in this review were done before 24 months. There are no studies providing guidance on appropriate patient selection when the $\mathrm{SCI}$ is more than 24 months old and when the muscle to be reanimated is paralyzed but not denervated. One author (van Zyl) has experience with preoperative electromyography in 18 cervical SCI patients (34 limbs). Ninety-five 
Table 3 Published nerve transfers in $\mathrm{SCl}$ in relation to function

\begin{tabular}{|l|l|l|l|l|l|l|l|}
\hline \multicolumn{2}{|l|}{ Elbow extension } & Thumb/finger extension & \multicolumn{2}{l|}{ Pinch and grasp } & \multicolumn{2}{l|}{ Wrist extension } \\
\hline Donor & Recipient & Donor & Recipient & Donor & Recipient & Donor & Recipient \\
\hline Teres minor & Triceps & Supinator & PIN & Distal ECRB & FPL & Brachialis & ECRL \\
\hline Posterior deltoid & Triceps & & & Brachialis & AIN & & \\
\hline Brachialis & Triceps & & & & & & \\
\hline
\end{tabular}

Abbreviations: AIN, anterior interosseous nerve; ECRB, extensor carpi radialis brevis; ECRL, extensor carpi radialis longus; FPL, flexor pollicis longus; $\mathrm{PIN}$, posterior interosseous nerve; $\mathrm{SCl}$, spinal cord injury.

potential recipient muscles for nerve transfer were examined, and fibrillation potentials, reflecting denervation, were present in 65.3\% (unpublished data). This finding indicates that there is often is a component of LMN injury to the nerve transfer target muscle and supports the argument that timing for nerve transfer surgery in SCI should be similar to that for plexus and peripheral nerve injury. ${ }^{15,20}$ Nerve transfers done late in the SCI population may yield results, but these are likely to be suboptimal. Early intervention with nerve transfers needs to be balanced against the chance of spontaneous recovery that can occur up to 12 months post injury in complete SCI and even later in incomplete injuries. ${ }^{21}$ Recovery of useful power in a paralyzed muscle more than 6 months post-complete SCI is unlikely. ${ }^{17}$ It remains to be elucidated in which cases a neural reconstruction remains reasonable even years later.

\section{Outcomes}

The primary goal of reconstructive upper limb surgery in SCI is to improve a patient's ability to function independently and perform activities of daily living (ADLs). Improvements in ADLs post-tendon transfer have been reported, ${ }^{23,24}$ with Canadian Occupational Performance Measurement (COPM) scores showing substantial improvement in both satisfaction and performance. ${ }^{25}$

The primary outcome reported in the nerve transfer cases in this review was strength grading according to the MRC scale. ${ }^{26}$ There was no consistent documentation or measurement tool used to report overall functional improvement in ADLs. As nerve transfer reconstructions become more commonplace in tetraplegia, functional outcome assessments such as the COPM, ${ }^{27}$ the Modified Action Research Arm Test, ${ }^{28}$ the Spinal Cord Independence Measure $^{29}$ must be applied to allow comparison with equivalent tendon transfer reconstructions and to measure the utility of nerve transfers. It is also imperative that we examine the cost-effectiveness of upper extremity reanimation through the use of nerve transfers to demonstrate the economic benefit of upper extremity reconstruction to the community and health administrators.

\section{Follow-up Duration}

The average follow-up time found in our review was 18 months post surgery, in contrast to a mean follow-up of
25 months in a systematic review of nerve transfers in adult upper brachial plexus injury. ${ }^{4}$ One case reported that a patient with tetraplegia who underwent brachialis to AIN transfer continued to gain both strength and functional control well after 15 months after surgery. ${ }^{30}$ The time to full maturation of nerve transfers in SCI is still unclear especially with the issue of whether recipient muscles are paralyzed as a result of a UMN or LMN or a combination of the two, clouding the picture.

\section{Combining Tendon Transfers and Nerve Transfers}

There are risks and benefits to both tendon and nerve transfers; surgeons and therapists need to carefully consider and individualize reconstruction options. The supinator to PIN nerve transfer arguably achieves superior hand opening compared with pronator to extensor digitorum communis tendon transfer but may still require an intrinsic reconstruction to flex the metacarpal and extend the interphalangeal joints, such as in the Alphabet procedure. ${ }^{25}$ The PIN nerve transfer reanimates not only the finger extensors but also the thumb extensors, extensor carpi ulnaris (ECU), and abductor pollicis longus (APL). It can potentially allow thumb extension independent of finger extension, and it allows good first web opening by reinnervating APL. Reanimation of the ECU centralizes the wrist for grasp. It remains to be evaluated whether the power attained by nerve transfers for pinch and grasp equals that attained in tendon transfer reconstructions, for example brachioradialis to FPL for pinch and the ECRL to the FDP for grasp, but the dexterity of the nerve transfer reconstruction may well provide some recompense for that.

Future practice will likely involve tendon and nerve transfer techniques performed in combination. The case reported by Bertelli and Ghizoni, restoring elbow extension, finger extension (metacarpal-phalangeal joint), thumb extension, and pinch, is a fine example of the potential restoration in upper limb function that can be achieved by combining tendon and nerve transfers in one operative stage. $^{11}$

\section{Target Population}

The future potential of this procedure will depend on its acceptance in the target population. Statistics show a predominance of SCI in young healthy males, who often 
in the early years postinjury, hold out hope for a "cure." A survey on tendon transfers showed that less than $50 \%$ of patients had been informed by their doctor about reconstructive upper limb surgery and only $9 \%$ of all participants in the study had gone ahead with the surgery. ${ }^{31}$ It has been reported that many patients delay pursuing surgical options that they perceive might potentially interfere with hand function should a cure for SCI be found. ${ }^{2}$ It is therefore important that the treating surgeon accurately explains the procedure to optimize uptake rate where outcome is likely to improve overall functional independence.

\section{Conclusion}

It is evident that to be able to draw accurate conclusions on the role of incorporating nerve transfers in the standard of care for tetraplegic patients, studies with larger numbers and longerterm follow-up need to be performed. Direct comparisons between outcomes in tendon and nerve transfer surgery need to be made. One such study is the current clinical trial (NCT01714349), entitled "Restoring Hand Function Using Nerve Transfers in Persons with Spinal Cord Injury." This is a 4-year interventional study that is currently in its first year recruiting participants for a single-nerve transfer, brachialis branch to the AIN. In addition, a pilot study (NCT01579604) is underway, which aims to look at the effectiveness of supinator to PIN transfer in five patients, operating at 6 to 9 months and reporting outcome at 24 months. Finally, The Victorian Spinal Cord Service at Austin Health in Melbourne, Australia, is conducting prospective case series (ACTRN12615000179538) to examine the outcomes and cost-benefit of nerve transfer surgery in tetraplegia.

Nerve transfers show promise as a reconstructive option in tetraplegia and provide novel options in cases not amenable to conventional tendon transfers such as restoring wrist extension-driven grip function in International Classification for Surgery of the Hand in Tetraplegia (ICSHT) ${ }^{32}$ group 0 patients by transfer of the brachialis to ECRL nerve or by restoring active hand opening in ICSHT group 4 tetraplegia using the supinator to PIN nerve transfer. A particularly exciting concept is combining nerve and muscle transfers into new algorithms, depending on a careful evaluation of individual factors, such as age, extent and nature of paralysis, functional needs, and time delay since SCI. Combining the advantages of both techniques will enable us to maximize arm and hand function in these severely handicapped patients as fast as possible.

It will be exciting to see the results of the registered clinical trials and to observe how upper limb nerve transfer surgery for tetraplegia evolves in the coming years.

Note

Portions of this work were presented in abstract/oral presentation form in proceedings at the Australian Hand Surgery Society Annual Scientific Meeting, Gold Coast, Queensland, 2014.
Conflicts of interest

None declared.

\section{References}

1 Bickenbach J. International Perspectives on Spinal Cord Injury. World Health Organization; 2013

2 Anderson KD. Targeting recovery: priorities of the spinal cordinjured population. J Neurotrauma 2004;21(10):1371-1383

3 Hentz VR, Leclercq C. Surgical Rehabilitation of the Upper Limb in Tetraplegia. New York, NY: WB Saunders; 2002

4 Yang LJ, Chang KW, Chung KC. A systematic review of nerve transfer and nerve repair for the treatment of adult upper brachial plexus injury. Neurosurgery 2012;71(2):417-429, discussion 429

5 Addas BM, Midha R. Nerve transfers for severe nerve injury. Neurosurg Clin N Am 2009;20(1):27-38, vi

6 Brown JM, Mackinnon SE. Nerve transfers in the forearm and hand. Hand Clin 2008;24(4):319-340, v

7 Benassy J. A case of transposition of the musculo-cutaneous nerve upon the median nerve. Paraplegia 1965;3(3):199-202

8 Kiwerski J. Recovery of simple hand function in tetraplegia patients following transfer of the musculo-cutaneous nerve into the median nerve. Paraplegia 1982;20(4):242-247

9 Krasuski M, Kiwerski J. An analysis of the results of transferring the musculocutaneous nerve onto the median nerve in tetraplegics. Arch Orthop Trauma Surg 1991;111(1):32-33

10 Bertelli JA, Ghizoni MF. Nerve transfers for elbow and finger extension reconstruction in midcervical spinal cord injuries. J Neurosurg 2015;122(1):121-127

11 Bertelli JA, Ghizoni MF. Single-stage surgery combining nerve and tendon transfers for bilateral upper limb reconstruction in a tetraplegic patient: case report. J Hand Surg Am 2013;38(7): 1366-1369

12 Brown JM. Nerve transfers in tetraplegia I: background and technique. Surg Neurol Int 2011;2:121

13 van Zyl N, Hahn JB, Cooper CA, Weymouth MD, Flood SJ, Galea MP. Upper limb reinnervation in C6 tetraplegia using a triple nerve transfer: case report. J Hand Surg Am 2014;39(9): 1779-1783

14 Bertelli JA, Ghizoni MF. Transfer of nerve branch to the brachialis to reconstruct elbow extension in incomplete tetraplegia: case report. J Hand Surg Am 2012;37(10):1990-1993

15 Bertelli JA, Ghizoni MF, Tacca CP. Transfer of the teres minor motor branch for triceps reinnervation in tetraplegia. J Neurosurg 2011; 114(5):1457-1460

16 Bertelli JA, Mendes Lehm VL, Tacca CP, Winkelmann Duarte EC, Ghizoni MF, Duarte H. Transfer of the distal terminal motor branch of the extensor carpi radialis brevis to the nerve of the flexor pollicis longus: an anatomic study and clinical application in a tetraplegic patient. Neurosurgery 2012;70(4):1011-1016, discussion 1016

17 Bertelli JA, Tacca CP, Ghizoni MF, Kechele PR, Santos MA. Transfer of supinator motor branches to the posterior interosseous nerve to reconstruct thumb and finger extension in tetraplegia: case report. J Hand Surg Am 2010;35(10):1647-1651

18 Bertelli JA, Tacca CP, Winkelmann Duarte EC, Ghizoni MF, Duarte H. Transfer of axillary nerve branches to reconstruct elbow extension in tetraplegics: a laboratory investigation of surgical feasibility. Microsurgery 2011;31(5):376-381

19 Mackinnon SE, Yee A, Ray WZ. Nerve transfers for the restoration of hand function after spinal cord injury. J Neurosurg 2012;117(1): 176-185

20 Fridén J, Gohritz A. Brachialis-to-extensor carpi radialis longus selective nerve transfer to restore wrist extension in tetraplegia: case report. J Hand Surg Am 2012;37(8):1606-1608 
21 Kakulas BA. Neuropathology: the foundation for new treatments in spinal cord injury. Spinal Cord 2004;42(10):549-563

22 Senjaya F, Midha R. Nerve transfer strategies for spinal cord injury. World Neurosurg 2013;80(6):319-326

23 Bryden AM, Peljovich AE, Hoyen HA, Nemunaitis G, Kilgore KL, Keith MW. Surgical restoration of arm and hand function in people with tetraplegia. Top Spinal Cord Inj Rehabil 2012;18(1): 43-49

24 Connolly SJ, Aubut JL, Teasell R, Jarus T. Enhancing upper extremity function with reconstructive surgery in persons with tetraplegia: a review of the literature. Top Spinal Cord Inj Rehabil 2007;13(1): 58-80

25 Reinholdt C, Fridén J. Outcomes of single-stage grip-release reconstruction in tetraplegia. J Hand Surg Am 2013;38(6): 1137-1144

26 MR C. Aids to the Examination of the Peripheral Nervous System. . Medical Research Council War Memorandum. London HMSO 45; 1976
27 Law M, Baptiste S, McColl M, Opzoomer A, Polatajko H, Pollock N. The Canadian occupational performance measure: an outcome measure for occupational therapy. Can J Occup Ther 1990;57(2):82-87

28 Lyle RC. A performance test for assessment of upper limb function in physical rehabilitation treatment and research. Int J Rehabil Res 1981;4(4):483-492

29 Catz A, Itzkovich M, Agranov E, Ring H, Tamir A. SCIM-spinal cord independence measure: a new disability scale for patients with spinal cord lesions. Spinal Cord 1997;35(12):850-856

30 Mackinnon SE, Ray WZ. Response. J Neurosurg 2013;118(3): 707-708

31 Anderson KD, Fridén J, Lieber RL. Acceptable benefits and risks associated with surgically improving arm function in individuals living with cervical spinal cord injury. Spinal Cord 2009;47(4): 334-338

32 McDowell CL, Moberg EA, House JH. The Second International Conference on Surgical Rehabilitation of the Upper Limb in Tetraplegia. J Hand Surg Am 1986;11:604-608 